\title{
A NOTE ON THE CROSSING NUMBERS OF 5-REGULAR GRAPHS ${ }^{1}$
}

\author{
ZHANGDONG OUYANG \\ Department of Mathematics \\ Hunan First Normal University \\ Changsha 410205, P.R. China \\ e-mail: oymath@163.com
}

\begin{abstract}
The crossing number $\operatorname{cr}(G)$ of a graph $G$ is the smallest number of edge crossings in any drawing of $G$. In this paper, we prove that there exists a unique 5-regular graph $G$ on 10 vertices with $\operatorname{cr}(G)=2$. This answers a question by Chia and Gan in the negative. In addition, we also give a new proof of Chia and Gan's result which states that if $G$ is a non-planar 5-regular graph on 12 vertices, then $\operatorname{cr}(G) \geq 2$.
\end{abstract}

Keywords: crossing number, 5-regular graph, drawing.

2010 Mathematics Subject Classification: 05C10, 05C62.

\section{REFERENCES}

[1] G. Chartrand and L. Lesniak, Graphs and Digraphs, 3rd Edition (Chapman \& Hall, New York, 1996).

[2] G.L. Chia and C.S. Gan, On crossing numbers of 5-regular graphs, in: J.-Y. Cai and C.K. Wong (Eds.), Computing and Combinatorics, Lecture Notes in Comput. Sci. 2387 (2002) 230-237. doi:10.1007/3-540-45655-4_26

[3] G.L. Chia and C.S. Gan, Minimal regular graphs with given girths and crossing numbers, Discuss. Math. Graph Theory 24 (2004) 223-237. doi:10.7151/dmgt.1227

\footnotetext{
${ }^{1}$ The work was supported by the National Natural Science Foundation of China (No. 11301169) and Hunan Provincial Natural Science Foundation of China (No. 2017JJ2055).
} 
[4] Z.D. Ouyang, J. Wang and Y.Q. Huang, The crossing number of join of the generalized Petersen graph $P(3,1)$ with path and cycle, Discuss. Math. Graph Theory 38 (2018) 351-370.

doi:10.7151/dmgt.2005

[5] M. Schaefer, Crossing Numbers of Graphs (CRC Press Inc., Boca Raton, Florida, 2017).

[6] Y.S. Yang, J.H. Lin and Y.J. Dai, Largest planar graphs and largest maximal planar graphs of diameter two, J. Comput. Appl. Math. 144 (2002) 349-358. doi:10.1016/S0377-0427(01)00572-6

Received 8 May 2018

Revised 7 January 2019

Accepted 7 January 2019 